\title{
Renewal reward processes with heavy-tailed inter-renewal times and heavy-tailed
} rewards

\author{
JOSHUA B. LEVY' and MURAD S. TAQQU ${ }^{2}$ \\ ${ }^{1}$ Department of Mathematics, Colgate University, 13 Oak Drive, Hamilton, NY 13346-1398, USA \\ ${ }^{2}$ Department of Mathematics, Boston University, 111 Cummington Street, Boston, MA 02215, \\ USA
}

It is well known that fractional Brownian motion can be obtained as the limit of a superposition of renewal reward processes with inter-renewal times that have infinite variance (heavy tails with exponent $\alpha$ ) and with rewards that have finite variance. We show here that if the rewards also have infinite variance (heavy tails with exponent $\beta$ ) then the limit $Z_{\beta}$ is a $\beta$-stable self-similar process. If $\beta \leqslant \alpha$, then $Z_{\beta}$ is the Lévy stable motion with independent increments; but if $\beta>\alpha$, then $Z_{\beta}$ is a stable process with dependent increments and self-similarity parameter $H=(\beta-\alpha+1) / \beta$.

Keywords: computer networks; infinite variance; self-similar processes; stable processes; telecommunications

\section{Introduction}

There has recently been a lot of interest in understanding why time series in computer Ethernet networks appear asymptotically self-similar (Leland et al. 1994). These time series measure deviations from the mean of the number of packets or bytes that are circulating through the network. A model based on on-off renewal processes was proposed in Willinger et al. (1997) and Taqqu et al. (1997) as a possible explanation. This model is a modification of a renewal reward model investigated by Mandelbrot (1969) and Taqqu and Levy (1986). In these papers it is fractional Brownian motion that appears in the limit. Fractional Brownian motion is a self-similar Gaussian process with stationary increments (a process $X(t)$ is said to be self-similar with index $H$ if, for all $a>0, a^{-H} X(a t)$ and $X(t)$ have identical finitedimensional distributions). The increments of fractional Brownian motion display 'long-range dependence' or 'long memory' because their correlations decrease like a power function and their spectral density explodes at the origin. Fractional Brownian motion is the best-known and most widely modelled self-similar process. But because it is Gaussian, its values do not differ greatly from the mean.

In this paper we will show that it is possible to obtain limits that are self-similar, with increments that are stationary, dependent and non-Gaussian. They will have a stable distribution with infinite variance. This means that the marginal distribution has a tail that 
decreases slowly, like a power function, and hence, that there is a much higher probability than in the Gaussian case that the increments differ greatly from their median value.

Our set-up is similar to that of Taqqu and Levy (1986). The renewal reward processes in that paper had infinite-variance inter-renewal times but finite-variance rewards. In this paper, both the inter-renewal times and the rewards are allowed to have infinite variance. More precisely, we suppose that the inter-renewal times are in the domain of attraction of a stable distribution with index $1<\alpha<2$ and the rewards are in the domain of attraction of a stable distribution with index $0<\beta<2$. The case $\beta<\alpha$ was considered in Levy and Taqqu (1987). The limit, in this case, was found to be the Lévy stable motion with index $\beta$ (we recover this result here). While this process is self-similar (with $H=1 / \beta$ ), has stationary increments and also infinite variances, its increments are independent. It is merely the infinite-variance counterpart of the Gaussian Brownian motion.

We consider here the more delicate case $\beta>\alpha$ and show that the limit is a symmetric stable process of index $\beta$, possesses stationary increments and is self-similar with index

$$
H=\frac{\beta-\alpha+1}{\beta} .
$$

Since $1<\alpha<\beta<2$, relation (1.1) implies

$$
H \in(1 / \beta, 1),
$$

$\beta$ being the index of stability of the limiting process. Observe that in the finite-variance case $(\beta=2)$, relation $(1.1)$ reduces to the familiar $H=(3-\alpha) / 2 \in\left(\frac{1}{2}, 1\right)$ which appears in connection with fractional Brownian motion.

Let us be more specific. The inter-renewal times are modelled by a sequence $U_{k}$, $k=1,2, \ldots$, of positive integer-valued independently and identically distributed (i.i.d.) random variables attracted to the stable domain with index $1<\alpha \leqslant 2(\alpha>1$ ensures that $U_{k}$ has a finite mean). The rewards are given by another i.i.d. sequence $W_{k}, k=0,1, \ldots$ of real-valued random variables belonging to the stable domain of index $0<\beta \leqslant 2$. $\left\{U_{k}\right\}$ and $\left\{W_{k}\right\}$ are assumed to be independent. A renewal-reward process $W=\{W(t), t=0,1, \ldots\}$ is constructed by assigning to each inter-renewal interval a corresponding reward that is constant throughout the interval (see Section 2 for a precise definition). We want to describe the asymptotic behaviour exhibited by large accumulations of these processes over long periods of time. Specifically, if $W_{m}(t), m=1,2, \ldots$, are i.i.d. copies of $W(t)$, then we consider the total reward process defined by

$$
W^{*}(T y, M)=\sum_{t=1}^{[T y]} \sum_{m=1}^{M} W_{m}(t),
$$

where $0 \leqslant y \leqslant 1, T=0,1, \ldots, M=1,2, \ldots$, and $[T y]$ stands for the greatest integer less than or equal to $T y$. $W^{*}$ can be viewed, for example, as the cumulative workload of $M$ workstations up to time $[T y]$. Our goal is to find the limiting behaviour of $W^{*}(T y, M)$, appropriately normalized as $M \rightarrow \infty$ followed by $T \rightarrow \infty$.

Two remarks are now in order. One concerns the order of the limits, the second the role of $y$. In Levy and Taqqu (1987), we investigated what happens when the limit is taken in 
the reverse order - first $T \rightarrow \infty$, then $M \rightarrow \infty$. We found that $W^{*}(T y, M)$, appropriately normalized, converges to Lévy stable motion with index $\beta$ if $\beta<\alpha$, and with index $\alpha$ if $\beta>\alpha$. We show in the present paper that, when $\beta>\alpha$, the order of the limits matters, because if we let $M \rightarrow \infty$ first, then $T \rightarrow \infty$, the limit process is not Lévy stable motion (which has independent increments) but is instead a stable self-similar process with dependent increments. The case where $T$ and $M$ tend jointly to infinity will be investigated in a separate paper.

Secondly, let us explain the role of $y$ in (1.3), which we take for convenience to be in the interval $[0,1]$, although in fact our results hold for any $y \geqslant 0$. The index $y$ corresponds to time and is introduced in order to characterize the behaviour of the stochastic process $W^{*}$ at large times. Consider, for example, two distinct values of $y$, say $y_{1}$ and $y_{2}$, and suppose that $T$ is large, so that $\left[T y_{1}\right]$ and $\left[T y_{2}\right]$ are large. The limit process we obtain characterizes, in particular, the behaviour of the random vector $\left(W^{*}\left(T y_{1}, M\right), W^{*}\left(T y_{2}, M\right)\right)$ for large $M$ and at large times $\left[T y_{1}\right]$ and $\left[T y_{2}\right]$, yielding information about the dependence structure of the cumulative workload at time $\left[T y_{1}\right]$ and the cumulative workload at time $\left[T y_{2}\right]$.

We now describe the limit process $Z_{\beta}$. We show, in Theorem 2.1 below, that when $M \rightarrow \infty$ followed by $T \rightarrow \infty, W^{*}(T y, M)$, renormalized, converges in the sense of finitedimensional distributions to a limiting process $\left\{Z_{\beta}(y), 0 \leqslant y \leqslant 1\right\}$. The process $Z_{\beta}$ is best described through its (finite-dimensional) characteristic functions as follows. Let $0 \leqslant y_{1} \leqslant y_{2} \leqslant \ldots \leqslant y_{d} \leqslant 1$ be $d$ time points and let $\theta_{1}, \theta_{2}, \ldots, \theta_{d}$ be arbitrary real numbers. For convenience set $\boldsymbol{y}=\left(y_{1}, \ldots, y_{d}\right)$ and $\boldsymbol{\theta}=\left(\theta_{1}, \ldots, \theta_{d}\right)$. Recall that $Z_{\beta}(\boldsymbol{y})$ is called a symmetric stable process of index $\beta$ if its finite-dimensional characteristic functions can be expressed as

$$
\mathrm{E} \exp \left\{\mathrm{i} \sum_{j=1}^{d} \theta_{j} Z_{\beta}\left(y_{j}\right)\right\}=\exp \left\{-\sigma^{\beta}(\boldsymbol{\theta}, \boldsymbol{y})\right\}
$$

where

$$
\sigma^{\beta}(\boldsymbol{\theta}, \boldsymbol{y})=\int_{E}\left|\sum_{j=1}^{d} \theta_{j} f_{j}(\xi, \boldsymbol{y})\right|^{\beta} m(\mathrm{~d} \xi) .
$$

Here $f_{1}, \ldots, f_{d}$ are functions defined on a measure space $(\mathrm{E}, \mathscr{E}, m)$ satisfying $\int_{E}\left|f_{j}(\xi, \boldsymbol{y})\right|^{\beta} m(\mathrm{~d} \xi)<\infty, j=1, \ldots, d$ (see Samorodnitsky and Taqqu 1994, p. 114). The functions $f_{1}, \ldots, f_{d}$ may depend on $\boldsymbol{y}$.

When $d=1$, for example (1.4) reduces to

$$
\mathrm{Ee}^{\mathrm{i} \theta Z_{\beta}\left(y_{1}\right)}=\mathrm{e}^{-|\theta|^{\beta} \sigma_{0}^{\beta}\left(y_{1}\right)},
$$

where $\sigma_{0}\left(y_{1}\right)=\left(\int_{E}\left|f\left(\xi, y_{1}\right)\right|^{\beta} m(\mathrm{~d} \xi)\right)^{1 / \beta}$ is the scale parameter of the symmetric stable random variable $Z_{\beta}\left(y_{1}\right)$.

When $d>1$, the function $\sigma(\boldsymbol{\theta}, \boldsymbol{y})$ not only characterizes the scale parameters of the marginal distributions of $Z_{\beta}\left(y_{1}\right), \ldots, Z_{\beta}\left(y_{d}\right)$, but also determines their dependence structure. 
We show in Theorem 2.1 that when $0<\beta \leqslant \alpha<2(1<\alpha<2)$, the limit process $Z_{\beta}(y)$ satisfies (1.4) with

$$
\sigma^{\beta}(\boldsymbol{\theta}, \boldsymbol{y})=C \sum_{j=1}^{d}\left|\phi_{j}\right|^{\beta}\left(y_{j}-y_{j-1}\right),
$$

where $C>0$ is a constant specified in the theorem and where

$$
\phi_{j}=\theta_{j}+\theta_{j+1}+\cdots+\theta_{d} \text {. }
$$

The process $Z_{\beta}(y)$ then has independent increments because its finite-dimensional characteristic functions factor:

$$
\begin{aligned}
\mathrm{E}\left\{\operatorname{expi} \sum_{j=1}^{d} \phi_{j}\left(Z_{\beta}\left(y_{j}\right)-Z_{\beta}\left(y_{j-1}\right)\right)\right\} & =\mathrm{E}\left\{\operatorname{expi} \sum_{j=1}^{d} \theta_{j} Z_{\beta}\left(y_{j}\right)\right\} \\
& =\exp \left\{-C \sum_{j=1}^{d}\left|\phi_{j}\right|^{\beta}\left(y_{j}-y_{j-1}\right)\right\} \\
& =\prod_{j=1}^{d} \exp \left\{-C\left|\phi_{j}\right|^{\beta}\left(y_{j}-y_{j-1}\right)\right\} \\
& =\prod_{j=1}^{d} \mathrm{E} \exp \left\{\mathrm{i} \phi_{j}\left(Z_{\beta}\left(y_{j}\right)-Z_{\beta}\left(y_{j-1}\right)\right\} .\right.
\end{aligned}
$$

$Z_{\beta}(y)$ has also stationary increments since adding a constant $h$ to each $y_{j}$ does not change the finite-dimensional characteristic functions of the increments. Therefore the process $\left\{Z_{\beta}(y)\right\}$ is (symmetric) Lévy stable motion with index $\beta$ when $\beta \leqslant \alpha$.

When $2>\beta>\alpha>1$, however, the limit $Z_{\beta}(y)$ is a different process. We show in Theorem 2.1 that, in this case, the limit process $Z_{\beta}(y)$ satisfies (1.4) with

$$
\sigma^{\beta}(\boldsymbol{\theta}, \boldsymbol{y})=C_{\beta}^{-1}(I(\boldsymbol{\theta}, \boldsymbol{y})+J(\boldsymbol{\theta}, \boldsymbol{y})),
$$

where

$$
C_{\beta}=\left(\int_{0}^{\infty} \frac{\sin x}{x^{\beta}} \mathrm{d} x\right)^{-1}=\frac{1-\beta}{\Gamma(2-\beta) \cos (\pi \beta / 2)},
$$

and

$$
\begin{gathered}
I(\boldsymbol{\theta}, \boldsymbol{y})=\int_{0}^{\infty} \mu^{-1}\left|\sum_{j=1}^{d} \theta_{j}\left(y_{j} \wedge x\right)\right|^{\beta} x^{-\alpha} \mathrm{d} x \\
J(\boldsymbol{\theta}, \boldsymbol{y})=\int_{0}^{\infty} \int_{0}^{\infty} \mu^{-1}\left|\sum_{j=1}^{d} \theta_{j}\left(y_{j} \wedge u-x\right)_{+}\right|^{\beta} \alpha(u-x)_{+}^{-\alpha-1} \mathrm{~d} u \mathrm{~d} x .
\end{gathered}
$$


(As usual, $(\cdot)_{+}=\max (\cdot, 0)$ ).

The process $Z_{\beta}(y)$ is then stable with index $\beta$. It is clearly self-similar with index $H=(\beta-\alpha+1) / \beta$ since $I(\boldsymbol{\theta}, a \boldsymbol{y})=a^{\beta-\alpha+1} I(\boldsymbol{\theta}, \boldsymbol{y})$ and $J(\boldsymbol{\theta}, a \boldsymbol{y})=a^{\beta-\alpha+1} J(\boldsymbol{\theta}, \boldsymbol{y})$. The stationarity of the increments, which is established in Section 6 , is not as obvious.

The paper is structured as follows. In Section 2, we present the basic assumptions and state the main result, Theorem 2.1, which includes the cases $0<\beta<\alpha$ and $\beta=\alpha$ as well as the case $\beta>\alpha$ described above. This theorem is a consequence of two others that are proved in Sections 3 and 4, respectively. Their proofs make use of a number of key propositions which are discussed in Section 5. The stationarity of the increments is established in Section 6.

\section{Assumptions and main results}

We start with the basic assumptions:

Assumption 1. The reward sequence $\left\{W_{k}\right\}, k=0,1, \ldots$, is i.i.d., symmetric and has heavy tails, that is, there exist an index $0<\beta<2$ and a slowly varying function $g(x)$ at infinity, such that

$$
P\left(\left|W_{0}\right|>x\right) \sim x^{-\beta} g(x) \text { as } x \rightarrow \infty
$$

Assumption 2. The inter-renewal times $\left\{U_{k}\right\}, k=1,2, \ldots$, are i.i.d. with range the positive integers and $P\left(U_{1}=x\right) \sim \alpha x^{-\alpha-1} h(x)$, where $1<\alpha<2$ and $h$ is slowly varying at infinity. Let $\mu=\mathrm{E} U_{1}$, which is finite, and let $U$ have the distribution of $U_{k}$.

Assumption 3. $\left\{W_{k}, k \geqslant 0\right\}$ and $\left\{U_{k}, k \geqslant 1\right\}$ are independent.

Assumption 1 implies that $W_{0}$ is in the domain of attraction of a symmetric stable random variable of index $0<\beta<2$, that is, there exists a sequence of constants $A_{n}(\beta)$ such that

$$
\mathscr{B}-\lim _{n \rightarrow \infty} A_{n}(\beta)^{-1} \sum_{k=1}^{n} W_{k} \stackrel{\mathscr{D}}{=} \Lambda_{\beta},
$$

where $\mathscr{B}$ and $\stackrel{\mathscr{P}}{=}$ refer, respectively, to convergence and equality in distribution. The normalization constants are $A_{n}(\beta)=n^{1 / \beta} L_{g}(n)$, where $L_{g}$ is a slowly varying function satisfying, for $x \neq 0$,

$$
\lim _{n \rightarrow \infty} L_{g}(n)^{-\beta} g\left(n^{1 / \beta} L_{g}(n)|x|\right)=1 .
$$

The stable random variable $\Lambda_{\beta}$ satisfies $P\left(\left|\Lambda_{\beta}\right|>x\right) \sim x^{-\beta}$ as $x \rightarrow \infty$, and its distribution is totally characterized by the index $\beta$.

We could have assumed $W_{0}$ to be non-symmetric for $\beta \neq 1$, that is, $P\left(W_{0} \leqslant-x\right) \sim$ $c^{-} x^{-\beta} g(x)$ and $P\left(W_{0} \geqslant x\right) \sim c^{+} x^{-\beta} g(x)$, and to satisfy, for centring purposes, $\mathrm{E} W_{0}=0$ for $1<\beta<2$. This situation is more realistic in the setting of telecommunications. In this case 
$\Lambda_{\beta}$ is skewed as well and satisfies $P\left(\Lambda_{\beta} \leqslant-x\right) \sim c^{-} x^{-\beta}$ and $P\left(\Lambda_{\beta}>x\right) \sim c^{+} x^{-\beta}$ as $x \rightarrow \infty$. It is for convenience' sake that we assume $W_{0}$ symmetric here. Results for $W_{0}$ skewed will be presented in a subsequent paper.

Similarly, Assumption 2 implies $P(U>x) \sim x^{-\alpha} h(x)$, and hence there exists a sequence of constants $B_{n}(\alpha)=n^{1 / \alpha} L_{h}(n)$ such that

$$
\mathscr{B}-\lim _{n \rightarrow \infty} B_{n}^{-1}(\alpha) \sum_{k=1}^{n}\left(U_{k}-\mu\right) \stackrel{\mathscr{D}}{=} \Lambda_{\alpha}^{+},
$$

where $L_{h}$ and $h$ are related as in (2.2), with $g$ replaced by $h$. Since $U_{1}$ is positive, the stable random variable $\Lambda_{\alpha}^{+}$is totally skewed to the right and satisfies $P\left(\Lambda_{\alpha}^{+}>x\right) \sim x^{-\alpha}$ and $P\left(\Lambda_{\alpha}^{+} \leqslant-x\right)=o\left(x^{-\alpha}\right)$, as $x \rightarrow \infty$.

We now turn to the construction of the total reward process. The sequence of interrenewal times defines a renewal process $\left\{S_{k}\right\}, k=0,1, \ldots$, by

$$
S_{k}=S_{0}+\sum_{j=1}^{k} U_{j}, \quad k \geqslant 1
$$

To make it stationary, we let

$$
P\left(S_{0}=x\right)=\mu^{-1} P(U>x), \quad x=0,1, \ldots .
$$

Relations (2.4) and (2.3) imply that $\left\{S_{k}, k \geqslant 0\right\}$ is a stationary renewal process.

The associated renewal-reward process $\{W(t), t=0,1, \ldots\}$ equals $W_{k}$ when $t$ is in the $k$ th inter-renewal interval. More precisely,

$$
W(t)=\sum_{k=0}^{t} W_{k} I\left(S_{k-1}<t \leqslant S_{k}\right), \text { which equals } W_{k} \text { if } S_{k-1}<t \leqslant S_{k},
$$

with $S_{-1} \equiv 0$. In particular, $W(0)=0$. The cumulative reward process up through time $T$ is given by $W^{*}(0)=0$ and

$$
W^{*}(T)=\sum_{t=1}^{T} W(t), T=1,2, \ldots
$$

Now let $\left\{W_{m}(t), t \geqslant 0\right\}, m=1,2, \ldots$, be i.i.d. copies of the process $W(t)$ and, similarly, $\left\{W_{m}^{*}(T), T \geqslant 0\right\}$ be i.i.d. copies of the process $W^{*}(T)$. Define the total reward process $W^{*}(T y, M), 0 \leqslant y \leqslant 1, T=0,1, \ldots$ and $M=1,2, \ldots$, by

$$
W^{*}(T y, M)=\sum_{m=1}^{M} W_{m}^{*}(T y)=\sum_{t=1}^{[T y]} \sum_{m=1}^{M} W_{m}(t) .
$$

We wish to find out how $W^{*}(T y, M)$, appropriately normalized, behaves as $M \rightarrow \infty$ and then $T \rightarrow \infty$. To express the normalization factors, introduce 


$$
a_{T} \equiv a_{T}(\alpha, \beta)= \begin{cases}T, & \beta<\alpha \text { or } \beta=\alpha \text { and } \sum_{x=1}^{\infty} x^{-1} h(x)<\infty, \\ T \int_{1}^{T} x^{-1} h(x) \mathrm{d} x, & \beta=\alpha \text { and } \sum_{x=1}^{\infty} x^{-1} h(x)=\infty, \\ T^{\beta-\alpha+1} h(T), & \beta>\alpha,\end{cases}
$$

where $h$ is the slowly varying function in Assumption 2. Also let $L_{g}$ be the slowly varying function in (2.2). A stochastic process is $H$-sssi if it is self-similar with index $H$ and has stationary increments (see Samorodnitsky and Taqqu (1994)). The following theorem provides the results for the cases $\beta<\alpha, \beta=\alpha$ and $\beta>\alpha$.

Theorem 2.1. Let Assumptions 1-3 hold and $W^{*}(T y, M)$ be defined by (2.7) and $a_{T}$ by (2.8). Then

$$
\mathscr{D}-\lim _{T \rightarrow \infty} \lim _{M \rightarrow \infty}\left[a_{T}^{1 / \beta} M^{1 / \beta} L_{g}(M)\right]^{-1} W^{*}(T y, M) \stackrel{\mathscr{D}}{=} Z_{\beta}(y), \quad 0 \leqslant y \leqslant 1,
$$

where $Z_{\beta} \equiv Z_{\beta}(y)$ is a symmetric $\beta$-stable $H$-self-similar process with stationary increments (H-sssi process) with

$$
H=\frac{(\beta-\alpha)_{+}+1}{\beta}= \begin{cases}1 / \beta, & \beta \leqslant \alpha \\ (\beta-\alpha+1) / \beta, & \beta>\alpha .\end{cases}
$$

$Z_{\beta}(0)=0$ and $Z_{\beta}$ is $\beta$-stable Lévy motion if and only if $\beta \leqslant \alpha$. Here $\mathscr{B}$ and $\stackrel{\mathscr{P}}{=}$ refer, respectively, to convergence and equality of the finite-dimensional distributions.

To characterize the finite-dimensional distributions of $Z_{\beta}$, consider any $\boldsymbol{\theta}=\left(\theta_{1}, \ldots\right.$, $\left.\theta_{d}\right) \in \mathbb{R}^{d}, \boldsymbol{y}=\left(y_{1}, \ldots, y_{d}\right) \in[0,1]^{d}, 0=y_{0} \leqslant y_{1} \leqslant \ldots \leqslant y_{d} \leqslant 1$. Then

$$
\mathrm{E} \exp \left\{\mathrm{i} \sum_{j=1}^{d} \theta_{j} Z_{\beta}\left(y_{j}\right)\right\}=\exp \left\{-\sigma^{\beta}(\boldsymbol{\theta}, \boldsymbol{y})\right\}
$$

where

$$
\sigma^{\beta}(\boldsymbol{\theta}, \boldsymbol{y})= \begin{cases}C_{\beta}^{-1}\left\{\mu^{-1} E U^{\beta} \sum_{i=1}^{d}\left|\phi_{i}\right|^{\beta}\left(y_{i}-y_{i-1}\right)\right\}, & \beta<\alpha \text { or } \beta=\alpha \text { and } \sum_{x=1}^{\infty} x^{-1} h(x)<\infty \\ C_{\beta}^{-1}\left\{\mu^{-1} \sum_{i=1}^{d}\left|\phi_{i}\right|^{\beta}\left(y_{i}-y_{i-1}\right)\right\}, & \beta=\alpha \text { and } \sum_{x=1}^{\infty} x^{-1} h(x)=\infty, \\ C_{\beta}^{-1}\{I(\boldsymbol{\theta}, \boldsymbol{y})+J(\boldsymbol{\theta}, \boldsymbol{y})\}, & \beta>\alpha .\end{cases}
$$

Here

$$
\begin{gathered}
\phi_{i}=\sum_{j=1}^{d} \theta_{j}, \\
C_{\beta}=\left(\int_{0}^{\infty} \frac{\sin x}{x^{\beta}} \mathrm{d} x\right)^{-1}= \begin{cases}\frac{1-\beta}{\Gamma(2-\beta) \cos (\pi \beta / 2)} & \text { if } \beta \neq 1 \\
2 / \pi & \text { if } \beta=1,\end{cases}
\end{gathered}
$$

and $I(\boldsymbol{\theta}, \boldsymbol{y})$ and $J(\boldsymbol{\theta}, \boldsymbol{y})$ are given by (1.6). 
When $\beta \leqslant \alpha$, the form of the characteristic function of $Z_{\beta}$ clearly identifies $Z_{\beta}$ with the symmetric Lévy stable motion with index $\beta$, which has independent increments. When $\beta>\alpha$, the increments are dependent and $Z_{\beta}$ is then no longer Lévy stable motion.

Observe that the expression for $\sigma^{\beta}(\boldsymbol{\theta}, \boldsymbol{y})$ in (2.12) contains the term $\mathrm{E} U^{\beta}$ when $\beta<\alpha$ and does not contain it if $\beta=\alpha$ and $\sum_{x=1}^{\infty} x^{-1} h(x)=\infty$. In the latter case $\mathrm{E} U^{\beta}=\mathrm{E} U^{\alpha}=\sum_{x=1}^{\infty} x^{\beta} P[U=x]=\sum_{x=1}^{\infty} x^{-1} h(x)=\infty$. The potential contribution of this term is compensated by the presence of the integral $\int_{1}^{T} x^{-1} h(x) \mathrm{d} x$ in the normalization factor $a_{T}$ in (2.8).

The proof of Theorem 2.1 is in two parts. The theorem follows from Theorems 2.2 $(M \rightarrow \infty)$ and $2.3(T \rightarrow \infty)$ stated below.

Theorem 2.2. Suppose Assumptions 1-3 hold. Then for each fixed $T=0,1, \ldots$,

$$
\mathscr{B}-\lim _{M \rightarrow \infty}\left[M^{1 / \beta} L_{g}(M)\right]^{-1} W^{*}(T y, M) \stackrel{\mathscr{P}}{=} Z_{\beta, T}(y), \quad 0 \leqslant y \leqslant 1 .
$$

$Z_{\beta, T} \equiv Z_{\beta, T}(y)$ is a symmetric $\beta$-stable process with $Z_{\beta, T}(0)=0$. For any $\boldsymbol{\theta}=\left(\theta_{1}, \ldots, \theta_{d}\right)$ $\in \mathbb{R}^{d}$ and $\boldsymbol{y}=\left(y_{1}, \ldots, y_{d}\right) \in[0,1]^{d}, \quad 0=y_{0} \leqslant y_{1} \leqslant \cdots \leqslant y_{d} \leqslant 1, \quad \sum_{i=1}^{d} \theta_{i} Z_{\beta, T}\left(y_{i}\right)$ is symmetric stable with index $\beta$ and scale parameter $\sigma_{T}(\boldsymbol{\theta}, \boldsymbol{y})$ satisfying

$$
\sigma_{T}^{\beta}(\boldsymbol{\theta}, \boldsymbol{y})=C_{\beta}^{-1} \mathrm{E} \sum_{k=0}^{\infty}\left|\sum_{j=1}^{d} \theta_{j}\left(\left[T y_{j}\right] \wedge S_{k}-S_{k-1}\right)_{+}\right|^{\beta} .
$$

The conclusion of Theorem 2.2 holds, in fact, for any non-decreasing sequence of random variables $S_{k}, k \geqslant-1$, with $S_{-1}=0$.

Observe that the process $Z_{\beta, T}(y)$ has neither stationary nor independent increments and is not self-similar.

Assumption 2 is used in the next theorem.

Theorem 2.3. If $a_{T}$ is given by (2.8) and $Z_{\beta, T}(y), 0 \leqslant y \leqslant 1$, is as in Theorem 2.2, then

$$
\mathscr{B}-\lim _{T \rightarrow \infty} a_{T}^{-1 / \beta} Z_{\beta, T}(y) \stackrel{\mathscr{D}}{=} Z_{\beta}(y), \quad 0 \leqslant y \leqslant 1,
$$

where $Z_{\beta}$ is the limit process of Theorem 2.1.

We prove Theorem 2.2 in Section 3 and Theorem 2.3 in Section 4.

\section{Proof of Theorem 2.2}

We shall use the following lemma.

Lemma 3.1. Suppose $\left\{W_{k}\right\}$ and $\left\{Y_{k}\right\}, k=1,2, \ldots$, are independent of each other; $\left\{W_{k}\right\}$ is i.i.d. and satisfies $P\left(\left|W_{1}\right|>x\right) \sim x^{-\beta} g(x)$ as $x \rightarrow \infty$ with $g$ slowly varying at infinity; and $\left\{Y_{k}\right\}$ is bounded a.s. and $K$ is an almost surely bounded positive integer-valued random 
variable. Then $\sum_{k=1}^{K} Y_{k} W_{k} \in \mathscr{D}(\beta)$, in particular, as $x \rightarrow \infty$,

$$
P\left(\left|\sum_{k=1}^{K} Y_{k} W_{k}\right|>x\right) \sim \mathrm{E}\left[\sum_{k=1}^{K}\left|Y_{k}\right|^{\beta}\right] x^{-\beta} g(x) .
$$

To verify this lemma, condition on $\left\{K, Y_{k}, k=1, \ldots, K\right\}$ and use the fact that $P\left(\left|\sum_{k=1}^{K_{0}} y_{k} W_{k}\right|>x\right) \sim \sum_{k=1}^{K_{0}}\left|y_{k}\right|^{\beta} x^{-\beta} g(x)$ for fixed constants $K_{0}$ and $y_{1}, \ldots, y_{K_{0}}$.

In order to prove Theorem 2.2, observe that the reward accumulated in the interval $S_{k-1}<t \leqslant S_{k}$ is $\left(S_{k}-S_{k-1}\right) W_{k}$, by (2.5). Now introduce the renewal function $K(T)$, namely, the total number of renewals up through time $T$. Rewriting the cumulative reward $W^{*}(T)$ in (2.6), using (2.3) and $K(T)$, we obtain

$$
\begin{aligned}
W^{*}(T) & =\left[S_{0} W_{0}+\sum_{k=1}^{K(T)-1} U_{k} W_{k}+\left(T-S_{K(T)-1}\right) W_{K(T)}\right] I\left(S_{0} \leqslant T\right)+T W_{0} I\left(S_{0}>T\right) \\
& =\left(T \wedge S_{0}\right) W_{0}+\sum_{k=1}^{K(T)}\left(T \wedge S_{k}-S_{k-1}\right)_{+} W_{k} I\left(S_{0} \leqslant T\right) \\
& =\sum_{k=0}^{K(T)}\left(T \wedge S_{k}-S_{k-1}\right)_{+} W_{k}=\sum_{k=0}^{\infty}\left(T \wedge S_{k}-S_{k-1}\right)_{+} W_{k},
\end{aligned}
$$

since $S_{-1} \equiv 0, S_{0}>T \Leftrightarrow K(T)=0 \Rightarrow\left(T \wedge S_{k}-S_{k-1}\right)_{+}=\left(T-S_{k-1}\right)_{+}=0$ for $k \geqslant 1$, and $k \geqslant K(T)+1 \Leftrightarrow S_{k-1}>T \Rightarrow\left(T \wedge S_{k}-S_{k-1}\right)_{+}=0$.

Since $\left(\left[T y_{j}\right] \wedge S_{k}-S_{k-1}\right)_{+}$is bounded by $T$, Lemma 3.1 applies. Theorem 2.2 follows because the $W_{m}^{*}(T)$ are i.i.d. copies of $W^{*}(T)$.

\section{Proof of Theorem 2.3}

To prove (2.17), it is sufficient to verify $\sum_{i=1}^{d} \theta_{i} Z_{\beta, T}\left(y_{i}\right) \stackrel{\mathscr{B}}{\rightarrow} \sum_{i=1}^{d} \theta_{i} Z_{\beta}\left(y_{i}\right)$ as $T \rightarrow \infty$ for any $\boldsymbol{\theta}=\left(\theta_{1}, \ldots, \theta_{d}\right) \in \mathbb{R}^{d}$ and $\boldsymbol{y}=\left(y_{1}, \ldots, y_{d}\right) \in[0,1]^{d}$ with $\theta_{j} \neq 0, j=1, \ldots, d$ and $0<$ $y_{1}<\cdots<y_{d} \leqslant 1$. Set

$$
y_{0}=0 \quad \text { and } \quad y_{d+1}=\infty .
$$

It suffices, therefore, to prove

$$
\lim _{T \rightarrow \infty} a_{T}^{-1} \sigma_{T}^{\beta}(\boldsymbol{\theta}, \boldsymbol{y})=\sigma^{\beta}(\boldsymbol{\theta}, \boldsymbol{y})
$$

where $a_{T}$ is given by (2.8), $\sigma_{T}^{\beta}(\boldsymbol{\theta}, \boldsymbol{y})$ is given by (2.16), and $\sigma^{\beta}(\boldsymbol{\theta}, \boldsymbol{y})$ is given by (2.12) and (1.6). Set

$$
T_{j}=\left[T y_{j}\right] \text { for } j=1, \ldots d, \quad \text { and } \quad T_{d+1}=\infty,
$$

and write in (2.16), 


$$
\mathrm{E} \sum_{k=0}^{\infty}\left|\sum_{j=1}^{d} \theta_{j}\left(T_{j} \wedge S_{k}-S_{k-1}\right)_{+}\right|^{\beta}=I(T, \boldsymbol{\theta}, \boldsymbol{y})+J(T, \boldsymbol{\theta}, \boldsymbol{y}),
$$

where

$$
I(T, \boldsymbol{\theta}, \boldsymbol{y}):=\mathrm{E}\left|\sum_{j=1}^{d} \theta_{j}\left(T_{j} \wedge S_{0}\right)\right|^{\beta}
$$

and

$$
J(T, \boldsymbol{\theta}, \boldsymbol{y}):=\sum_{k=1}^{\infty} \mathrm{E}\left|\sum_{j=1}^{d} \theta_{j}\left(T_{j} \wedge S_{k}-S_{k-1}\right)_{+}\right|^{\beta} .
$$

The estimation of $\lim _{T \rightarrow \infty} I(T, \boldsymbol{\theta}, \boldsymbol{y})$ is done in Proposition 5.1 below.

Regarding $J(T, \boldsymbol{\theta}, \boldsymbol{y})$, write

$$
J(T, \boldsymbol{\theta}, \boldsymbol{y})=\sum_{k=1}^{\infty} J(T, \boldsymbol{\theta}, \boldsymbol{y}, k),
$$

where

$$
J(T, \boldsymbol{\theta}, \boldsymbol{y}, k)=\mathrm{E}\left|\sum_{j=1}^{d} \theta_{j}\left(T_{j} \wedge S_{k}-S_{k-1}\right)_{+}\right|^{\beta} .
$$

Setting $N_{d}:=\{1,2, \ldots, d\}, d \geqslant 1$, and $\mathscr{C}=\left\{\left(i_{1}, i_{2}\right) \in N_{d+1} \times N_{d+1}: i_{1} \leqslant i_{2}\right\}$, we can rewrite $J(T, \boldsymbol{\theta}, \boldsymbol{y}, k)$ as

$$
\begin{aligned}
J(T, \boldsymbol{\theta}, \boldsymbol{y}, k) & =\mathrm{E}\left|\sum_{b} \sum_{j=1}^{d} \theta_{j}\left(T_{j} \wedge S_{k}-S_{k-1}\right)_{+} I\left(T_{i_{1}-1}<S_{k-1} \leqslant T_{i_{1}}, T_{i_{2}-1}<S_{k} \leqslant T_{i_{2}}\right)\right|^{\beta} \\
& =\sum_{b} B_{T}\left(k, i_{1}, i_{2}\right),
\end{aligned}
$$

where $i_{1} \leqslant i_{2}$ and

$$
\begin{aligned}
B_{T}\left(k, i_{1}, i_{2}\right): & =\mathrm{E}\left|\sum_{j=1}^{d} \theta_{j}\left(T_{j} \wedge S_{k}-S_{k-1}\right)_{+} I\left(T_{i_{1}-1}<S_{k-1} \leqslant T_{i_{1}}, T_{i_{2}-1}<S_{k} \leqslant T_{i_{2}}\right)\right|^{\beta} \\
& =\sum_{x=T_{i_{1}-1}+1}^{T_{i_{1}}} \sum_{u=T_{i_{2}-1}+1-x}^{T_{i_{2}-x}}\left|\sum_{j=1}^{d} \theta_{j}\left(T_{j} \wedge(x+u)-x\right)_{+}\right|^{\beta} P\left(S_{k-1}=x\right) P(U=u),
\end{aligned}
$$

since $S_{k-1}$ is independent of $U_{k}$ and $U_{k} \stackrel{\mathscr{D}}{=} U$. Substituting back into (4.6) and then (4.6) back into (4.5), factoring out $P\left(S_{k-1}=x\right)$ and summing over $k$ to obtain $\sum_{k=1}^{\infty} P\left(S_{k-1}=x\right)=\mu^{-1}$, and then making the variable change $u \mapsto u+x$, gives 


$$
J(T, \boldsymbol{\theta}, \boldsymbol{y})=\sum_{\not{b}} \sum_{x=T_{i_{1}-1}+1}^{T_{i_{1}}} \sum_{u=T_{i_{2}-1}+1}^{T_{i_{2}}} \mu^{-1}\left|\sum_{j=1}^{d} \theta_{j}\left(T_{j} \wedge u-x\right)_{+}\right|^{\beta} P(U=u-x) .
$$

Dividing $\mathscr{A}$ into the subsets

$$
\begin{aligned}
& \mathscr{A}_{1}=\left\{\left(i_{1}, i_{2}\right) \in \mathscr{A}: i_{1}=i_{2}\right\}=\left\{\left(i_{1}, i_{2}\right): i_{1}=i_{2}=i, 1 \leqslant i \leqslant d+1\right\}, \\
& \mathscr{C}_{2}=\left\{\left(i_{1}, i_{2}\right) \in \mathscr{A}: i_{1}<i_{2}-1\right\}=\left\{\left(i_{1}, i_{2}\right): i_{1}<i_{2}-1=i, 1 \leqslant i \leqslant d\right\}, \\
& \mathscr{b}_{3}=\left\{\left(i_{1}, i_{2}\right) \in \mathscr{b}: i_{1}=i_{2}-1\right\}=\left\{\left(i_{1}, i_{2}\right): i_{1}=i_{2}-1=i, 1 \leqslant i \leqslant d\right\},
\end{aligned}
$$

we have

$$
J(T, \boldsymbol{\theta}, \boldsymbol{y})=J_{1}(T, \boldsymbol{\theta}, \boldsymbol{y})+J_{2}(T, \boldsymbol{\theta}, \boldsymbol{y})+J_{3}(T, \boldsymbol{\theta}, \boldsymbol{y}),
$$

where $J_{l}$ is $J$ with $A_{0}$ replaced by $\mathscr{C}_{l}, l=1,2,3$.

The estimation of $\lim _{T \rightarrow \infty} J_{l}(T, \boldsymbol{\theta}, \boldsymbol{y}), l=1,2,3$, is carried out in Propositions 5.2, 5.3 and 5.4 respectively (see Section 5 below). By substituting the results into (4.9), we obtain $\lim _{T \rightarrow \infty} J(T, \boldsymbol{\theta}, \boldsymbol{y})$. Combining this with $\lim _{T \rightarrow \infty} I(T, \boldsymbol{\theta}, \boldsymbol{y})$ in (4.2), we obtain $\lim _{T \rightarrow \infty} \mathrm{E} \sum_{k=0}^{\infty}\left|\sum_{j=1}^{d} \theta_{j}\left(T_{j} \wedge S_{k}-S_{k-1}\right)_{+}\right|^{\beta}$. In the case $\beta \leqslant \alpha, J_{1}(T, \boldsymbol{\theta}, \boldsymbol{y})$ dominates $J_{2}(T, \boldsymbol{\theta}, \boldsymbol{y}), \quad J_{3}(T, \boldsymbol{\theta}, \boldsymbol{y})$ and $I(T, \boldsymbol{\theta}, \boldsymbol{y})$, so $J_{1}$ alone provides the rate of growth $\mathrm{E} \sum_{k=0}^{\infty}\left|\sum_{j=1}^{d} \theta_{j}\left(T_{j} \wedge S_{k}-S_{k-1}\right)_{+}\right|^{\beta}$. In fact, if $\beta<\alpha$ or if $\beta=\alpha$ and $\sum_{x=1}^{\infty} x^{-1} h(x)<\infty$, we have

$$
\begin{aligned}
\mathrm{E} \sum_{k=1}^{\infty}\left|\sum_{j=1}^{d} \theta_{j}\left(T_{j} \wedge S_{k}-S_{k-1}\right)_{+}\right|^{\beta} & \sim J(T, \boldsymbol{\theta}, \boldsymbol{y}) \sim J_{1}(T, \boldsymbol{\theta}, \boldsymbol{y}) \\
& \sim \mu^{-1}\left(\mathrm{E} U^{\beta}\right) \sum_{i=1}^{d}\left|\phi_{i}\right|^{\beta}\left(y_{i}-y_{i-1}\right) T,
\end{aligned}
$$

and, if $\beta=\alpha$ and $\sum_{x=1}^{\infty} x^{-1} h(x)=\infty$, then

$$
\begin{aligned}
\mathrm{E} \sum_{k=1}^{\infty}\left|\sum_{j=1}^{d} \theta_{j}\left(T_{j} \wedge S_{k}-S_{k-1}\right)_{+}\right|^{\beta} & \sim J(T, \boldsymbol{\theta}, \boldsymbol{y}) \sim J_{1}(T, \boldsymbol{\theta}, \boldsymbol{y}) \\
& \sim \mu^{-1} \sum_{i=1}^{d}\left|\phi_{i}\right|^{\beta}\left(y_{i}-y_{i-1}\right) T \int_{1}^{T} x^{-1} h(x) \mathrm{d} x
\end{aligned}
$$

where $\int_{1}^{T} x^{-1} h(x) \mathrm{d} x$ tends to infinity, like a slowly varying function, but faster than $h(T)$.

In the case $\beta>\alpha$, each term $I(T, \boldsymbol{\theta}, \boldsymbol{y}), J_{1}(T, \boldsymbol{\theta}, \boldsymbol{y}), J_{2}(T, \boldsymbol{\theta}, \boldsymbol{y})$ and $J_{3}(T, \boldsymbol{\theta}, \boldsymbol{y})$ contributes the growth rate of $T^{\beta-\alpha+1} h(T)$. Since $I(\boldsymbol{\theta}, \boldsymbol{y})$ and $J(\boldsymbol{\theta}, \boldsymbol{y})$ are given by $(1.6)$, and (4.4), (4.8) and (4.9) imply

$$
J(\boldsymbol{\theta}, \boldsymbol{y})=J_{1}(\boldsymbol{\theta}, \boldsymbol{y})+J_{2}(\boldsymbol{\theta}, \boldsymbol{y})+J_{3}(\boldsymbol{\theta}, \boldsymbol{y}),
$$

we obtain 


$$
\mathrm{E} \sum_{k=0}^{\infty}\left|\sum_{j=1}^{d} \theta_{j}\left(T_{j} \wedge S_{k}-S_{k-1}\right)_{+}\right|^{\beta} \sim[I(\boldsymbol{\theta}, \boldsymbol{y})+J(\boldsymbol{\theta}, \boldsymbol{y})] T^{\beta-\alpha-1} h(T) .
$$

It follows, now, that (4.1) holds with $\sigma^{\beta}(\boldsymbol{\theta}, \boldsymbol{y})$ given by (2.12).

A particular consequence of (2.12) is the 'scaling' relation

$$
\sigma^{\beta}(\boldsymbol{\theta}, a \boldsymbol{y})=a^{\beta H} \sigma^{\beta}(\boldsymbol{\theta}, \boldsymbol{y}), \quad a>0,
$$

with $H$ defined in (2.10). This proves that $Z_{\beta}$ is self-similar with parameter $H$ since, for any $\gamma \in \mathbb{R}$ and $a>0$,

$$
\mathrm{E} \exp \left\{\mathrm{i} \gamma \sum_{j=1}^{d} \theta_{j} Z_{\beta}\left(a y_{j}\right)\right\}=\mathrm{E} \exp \left\{\mathrm{i} \gamma a^{H} \sum_{j=1}^{d} \theta_{j} Z_{\beta}\left(y_{j}\right)\right\} .
$$

Moreover, by Proposition 6.1 below, the process $Z_{\beta}$ has stationary increments. Relation (2.12) implies that when $\beta \leqslant \alpha$, it has independent increments, and therefore it is Lévy stable motion in this case.

\section{Propositions}

Let $\boldsymbol{\theta}=\left(\theta_{1}, \ldots, \theta_{d}\right)$ and $\boldsymbol{y}=\left(y_{1}, \ldots, y_{d}\right)$ be arbitrary. We assume without loss of generality that $\theta_{j} \neq 0, j=1, \ldots, d$ and $0<y_{1}<\cdots<y_{d} \leqslant 1$, and set $y_{0}=0$. The following propositions are used in the proof of Theorem 2.3.

Proposition 5.1. As $T \rightarrow \infty$,

$I(T, \boldsymbol{\theta}, \boldsymbol{y}) \sim \begin{cases}O(1), & \text { if } \beta<\alpha-1 \text { or if } \beta=\alpha-1 \text { and } \sum_{x=1}^{\infty} x^{-1} h(x)<\infty, \\ O\left(\int_{1}^{T} x^{-1} h(x) \mathrm{d} x\right), & \text { if } \beta=\alpha-1 \text { and } \sum_{x=1}^{\infty} x^{-1} h(x)=\infty, \\ I(\boldsymbol{\theta}, \boldsymbol{y}) T^{\beta-\alpha+1} h(T), & \text { if } \beta>\alpha-1,\end{cases}$

where

$$
I(\boldsymbol{\theta}, \boldsymbol{y}):=\int_{0}^{\infty} \mu^{-1}\left|\sum_{j=1}^{d} \theta_{j}\left(y_{j} \wedge x\right)\right|^{\beta} x^{-\alpha} \mathrm{d} x
$$

Remark. The term $I(T, \boldsymbol{\theta}, \boldsymbol{y})$ provides a non-negligible contribution to the limit in (2.9) only when $\beta>\alpha$.

Proof. From (4.3) and (2.4),

$$
I(T, \boldsymbol{\theta}, \boldsymbol{y})=\mathrm{E}\left|\sum_{j=1}^{d} \theta_{j}\left(T_{j} \wedge S_{0}\right)\right|^{\beta}=\mu^{-1} \sum_{x=0}^{\infty}\left|\sum_{j=1}^{d} \theta_{j}\left(T_{j} \wedge x\right)\right|^{\beta} P(U>x) .
$$


Suppose first that $\beta \leqslant \alpha-1$. Subdivide $\sum_{x=0}^{\infty}$ into $\sum_{x=1}^{T_{1}}$ and $\sum_{x=T_{1}+1}^{\infty}$ and call $I_{1}$ and $I_{2}$ the respective sums. Since $0 \leqslant x \leqslant T_{1}$ implies $T_{j} \wedge x=x$ for $j=1, \ldots, d$, we have

$$
I_{1}(T, \boldsymbol{\theta}, \boldsymbol{y})=\mu^{-1}\left|\sum_{j=1}^{d} \theta_{j}\right|^{\beta} \sum_{x=1}^{T_{1}} x^{\beta} P(U>x) .
$$

$I_{1}$ is 0 if $\sum_{j=1}^{d} \theta_{j}=0$. If $\sum_{j=1}^{d} \theta_{j} \neq 0$, then $I_{1}$ is $O(1)$ if $\beta<\alpha-1$ or if $\beta=\alpha-1$ and $\sum_{x=1}^{\infty} x^{-1} h(x)<\infty ; \quad I_{1}=O\left(\int_{1}^{T} x^{-1} h(x) \mathrm{d} x\right)$ if $\beta=\alpha-1$ and $\sum_{x=1}^{\infty} x^{-1} h(x)=\infty$. On the other hand,

$$
I_{2}(T, \boldsymbol{\theta}, \boldsymbol{y})= \begin{cases}o(1), & \text { if } \beta<\alpha-1 \text { or } \beta=\alpha-1 \text { and } \sum_{x=1}^{\infty} x^{-1} h(x)<\infty, \\ O(h(T)), & \text { if } \beta=\alpha-1 \text { and } \sum_{x=1}^{\infty} x^{-1} h(x)=\infty,\end{cases}
$$

using in the first case $T_{j} \wedge x \leqslant x$ and $\sum_{x=T_{1}+1}^{\infty} x^{\beta} P(U>x)=o(1)$, and using in the second case $\quad T_{j} \wedge x \leqslant T_{j} \leqslant T \quad$ and $\quad \sum_{x=T_{1}+1}^{\infty} P(U>x)=O\left(T^{1-\alpha} h(T)\right)$. Since $h(T)=$ $o\left(\int_{1}^{T} x^{-1} h(x) \mathrm{d} x\right)$, the term $I_{2}$ is negligible with respect to $I_{1}$ unless $\sum_{i=1}^{d} \theta_{i}=0$. This completes the proof in the case $\beta \leqslant \alpha-1$.

We now turn to the case $\beta>\alpha-1$. We first show that as $T \rightarrow \infty$,

$$
\tilde{I}(T, \boldsymbol{\theta}, \boldsymbol{y}):=\mu^{-1} \int_{0}^{\infty}\left|\sum_{j=1}^{d} \theta_{j}\left(T_{j} \wedge x\right)\right|^{\beta} P(U>x) \mathrm{d} x \sim I(\boldsymbol{\theta}, \boldsymbol{y}) T^{\beta-\alpha+1} h(T),
$$

by writing $\int_{0}^{\infty}=\int_{0}^{x_{0}}+\int_{x_{0}}^{\infty}$ and noting that the first integral is $O(1)$ while the second is asymptotic to $I(\boldsymbol{\theta}, \boldsymbol{y}) T^{\beta-\alpha+1} h(T)$.

Next, we show that

$$
I(T, \boldsymbol{\theta}, \boldsymbol{y})-\tilde{I}(T, \boldsymbol{\theta}, \boldsymbol{y})=o\left(T^{\beta-\alpha+1} h(T)\right) .
$$

By (5.3),

$$
\begin{aligned}
& I(T, \boldsymbol{\theta}, \boldsymbol{y})-\tilde{I}(T, \boldsymbol{\theta}, \boldsymbol{y}) \\
& \quad=\mu^{-1} \sum_{x=1}^{\infty} \int_{x-1}^{x}\left[\left|\sum_{j=1}^{d} \theta_{j}\left(T_{j} \wedge x\right)\right|^{\beta} P(U>x)-\left|\sum_{j=1}^{d} \theta_{j}\left(T_{j} \wedge t\right)\right|^{\beta} P(U>t)\right] \mathrm{d} t \\
& \quad=N_{1}(T, \boldsymbol{\theta}, \boldsymbol{y})+N_{2}(T, \boldsymbol{\theta}, \boldsymbol{y}),
\end{aligned}
$$

with

$$
N_{1}(T, \boldsymbol{\theta}, \boldsymbol{y})=\mu^{-1} \sum_{i=1}^{d+1} \sum_{x=T_{i-1}+1}^{T_{i}} \int_{x-1}^{x}\left[\left|\sum_{j=1}^{d} \theta_{j}\left(T_{j} \wedge x\right)\right|^{\beta}-\left|\sum_{j=1}^{d} \theta_{j}\left(T_{j} \wedge t\right)\right|^{\beta}\right] P(U>t) \mathrm{d} t
$$

and

$$
N_{2}(T, \boldsymbol{\theta}, \boldsymbol{y})=\mu^{-1} \sum_{i=1}^{d+1} \sum_{x=T_{i-1}+1}^{T_{i}} \int_{x-1}^{x}\left|\sum_{j=1}^{d} \theta_{j}\left(T_{j} \wedge x\right)\right|^{\beta}[P(U>x)-P(U>t)] \mathrm{d} t
$$


Using the inequality

$$
\left.|| a\right|^{\beta}-|b|^{\beta} \mid \leqslant \begin{cases}|a-b| & \text { if } 0<\beta \leqslant 1 \\ \beta|a-b|\left(|a|^{\beta-1}+|b|^{\beta-1}\right) & \text { if } 1<\beta\end{cases}
$$

for real numbers $a, b$, one can show $N_{1}(T, \boldsymbol{\theta}, \boldsymbol{y})=O\left(T^{(\beta-1)_{+}}\right)$. Moreover, one can also show that $N_{2}(T, \boldsymbol{\theta}, \boldsymbol{y})=O\left(\left[\int_{1}^{T} x^{-1} h(x) \mathrm{d} x\right] \vee T^{\beta-\alpha} h(T)\right)$. Combining these estimates for $N_{1}$ and $N_{2}$, one establishes (5.4). Consequently,

$$
I(T, \boldsymbol{\theta}, \boldsymbol{y}) \sim \tilde{I}(T, \boldsymbol{\theta}, \boldsymbol{y}) \sim I(\boldsymbol{\theta}, \boldsymbol{y}) T^{\beta-\alpha+1} h(T) .
$$

We now state the propositions for $J_{1}, J_{2}$ and $J_{3}$ and then outline their proofs. Recall that $J_{1}(T, \boldsymbol{\theta}, \boldsymbol{y}), J_{2}(T, \boldsymbol{\theta}, \boldsymbol{y})$, and $J_{3}(T, \boldsymbol{\theta}, \boldsymbol{y})$ are defined as $J$ in (4.7) with $\mathscr{A}$ replaced by $\mathscr{C}_{1}$, $\mathscr{A}_{2}, \mathscr{A}_{3}$, respectively (see (4.8)).

Proposition 5.2. As $T \rightarrow \infty$,

$J_{1}(T, \boldsymbol{\theta}, \boldsymbol{y}) \sim$

$$
\begin{cases}\mu^{-1}\left(\mathrm{E} U^{\beta}\right) \sum_{i=1}^{d}\left|\phi_{i}\right|^{\beta}\left(y_{i}-y_{i-1}\right) T, & \text { if } \beta<\alpha \text { or } \beta=\alpha \text { and } \sum_{x=1}^{\infty} x^{-1} h(x)<\infty, \\ \mu^{-1} \sum_{i=1}^{d}\left|\phi_{i}\right|^{\beta}\left(y_{i}-y_{i-1}\right) T \int_{1}^{T} x^{-1} h(x) \mathrm{d} x, & \text { if } \beta=\alpha \text { and } \sum_{x=1}^{\infty} x^{-1} h(x)=\infty, \\ J_{1}(\boldsymbol{\theta}, \boldsymbol{y}) T^{\beta-\alpha+1} h(T), & \text { if } \beta>\alpha,\end{cases}
$$

where

$$
J_{1}(\boldsymbol{\theta}, \boldsymbol{y}):=\sum_{i=1}^{d} \int_{y_{i-1}}^{y_{i}} \int_{y_{i-1}}^{y_{i}} H(\boldsymbol{\theta}, \boldsymbol{y} ; x, u) \mathrm{d} u \mathrm{~d} x
$$

and

$$
H(\boldsymbol{\theta}, \boldsymbol{y} ; x, u):=\mu^{-1}\left|\sum_{j=1}^{d} \theta_{j}\left(y_{j} \wedge u-x\right)_{+}\right|^{\beta} \alpha(u-x)_{+}^{-\alpha-1}
$$

Remark. Note that for $i_{1} \leqslant i_{2}, y_{i_{1}-1} \leqslant x \leqslant y_{i_{1}}, y_{i_{2}-1} \leqslant u \leqslant y_{i_{2}}$, we have

$$
\sum_{j=1}^{d} \theta_{j}\left(y_{j} \wedge u-x\right)_{+}=\sum_{j=1}^{i_{1}-1}+\sum_{j=i_{1}}^{i_{2}-1}+\sum_{j=i_{2}}^{d}=0+\sum_{j=i_{1}}^{i_{2}-1} \theta_{j}\left(y_{j}-x\right)+\phi_{i_{2}}(u-x)_{+} .
$$

Since, when $i_{1}=i_{2}=i$, this reduces to $\phi_{i}(u-x)_{+}, J_{1}(\boldsymbol{\theta}, \boldsymbol{y})$ defined in (5.6), can also be expressed as

$$
J_{1}(\boldsymbol{\theta}, \boldsymbol{y})=\mu^{-1} \alpha(\beta-\alpha)^{-1}(\beta-\alpha+1)^{-1} \sum_{i=1}^{d}\left|\phi_{i}\right|^{\beta}\left(y_{i}-y_{i-1}\right)^{\beta-\alpha+1} .
$$


Proposition 5.3. As $T \rightarrow \infty$,

$$
J_{2}(T, \boldsymbol{\theta}, \boldsymbol{y}) \sim J_{2}(\boldsymbol{\theta}, \boldsymbol{y}) T^{\beta-\alpha+1} h(T),
$$

where

$$
J_{2}(\boldsymbol{\theta}, \boldsymbol{y})=\sum_{1 \leqslant i_{1}<i_{2}-1 \leqslant d} \int_{y_{i_{1}-1}}^{y_{i_{1}}} \int_{y_{i_{2}-1}}^{y_{i_{2}}} H(\boldsymbol{\theta}, \boldsymbol{y} ; x, u) \mathrm{d} u \mathrm{~d} x
$$

and $H(\boldsymbol{\theta}, \boldsymbol{y} ; x, u)$ is given by (5.7).

Proposition 5.4. As $T \rightarrow \infty$,

$J_{3}(T, \boldsymbol{\theta}, \boldsymbol{y}) \sim \begin{cases}O(1), & \text { if } \beta<\alpha-1 \text { or if } \beta=\alpha-1 \text { and } \sum_{x=1}^{\infty} x^{-1} h(x)<\infty, \\ O\left(\int_{1}^{T} x^{-1} h(x) \mathrm{d} x\right), & \text { if } \beta=\alpha-1 \text { and } \sum_{x=1}^{\infty} x^{-1} h(x)=\infty, \\ J_{3}(\boldsymbol{\theta}, \boldsymbol{y}) T^{\beta-\alpha+1} h(T), & \text { if } \beta>\alpha-1,\end{cases}$

where

$$
J_{3}(\boldsymbol{\theta}, \boldsymbol{y}):=\sum_{i=1}^{d} \int_{y_{i-1}}^{y_{i}} \int_{y_{i}}^{y_{i+1}} H(\boldsymbol{\theta}, \boldsymbol{y} ; x, u) \mathrm{d} u \mathrm{~d} x
$$

and $H(\boldsymbol{\theta}, \boldsymbol{y} ; x, u)$ is defined by (5.7).

Remark. $J_{3}(T, \boldsymbol{\theta}, \boldsymbol{y})$ and $I(T, \boldsymbol{\theta}, \boldsymbol{y})$ are asymptotically proportional in the case $\beta>\alpha-1$.

In order to prove the propositions involving $J_{1}, J_{2}$ and $J_{3}$, we express $J(T, \boldsymbol{\theta}, \boldsymbol{y})$ in (4.7) in a more convenient manner. Suppose throughout that $i_{1} \leqslant i_{2}, T_{i_{1}-1}+1 \leqslant x \leqslant T_{i_{1}}$ and $T_{i_{2}-1}+1 \leqslant u \leqslant T_{i_{2}}$, as these constraints obtain in (4.7). Observe, now, that:

(i) for $j=1, \ldots, i_{1}-1$, we have $\left(T_{j} \wedge u-x\right)_{+}=\left(T_{j}-x\right)_{+}=0$;

(ii) for $j=i_{1}, \ldots, i_{2}-1$, we have $\left(T_{j} \wedge u-x\right)_{+}=T_{j}-x$;

(iii) for $j=i_{2}, \ldots, d$, we have $\left(T_{j} \wedge u-x\right)_{+}=(u-x)_{+}$.

Using the notation $\phi_{i}=\sum_{j=i}^{d} \theta_{j}$ and $\phi_{d+1}=0$, we obtain

$$
J(T, \boldsymbol{\theta}, \boldsymbol{y})=\sum_{\mathscr{b}} \sum_{x=T_{i_{1}-1}+1}^{T_{i_{1}}} \sum_{u=T_{i_{2}-1}+1}^{T_{i_{2}}} \mu^{-1}\left|\sum_{j=i_{1}}^{i_{2}-1} \theta_{j}\left(T_{j}-x\right)+\phi_{i_{2}}(u-x)_{+}\right|^{\beta} P(U=u-x) .
$$

To get $J_{1}, J_{2}$ and $J_{3}$, we replace $\mathscr{A}$ by $\mathscr{C}_{1}, \mathscr{A}_{2}$ and $\mathscr{C}_{3}$, respectively (see (4.8)).

Proof of Proposition 5.2. Since $J_{1}$ is $J$ with $\mathscr{A}$ replaced by $\mathscr{A}_{1}=\left\{\left(i_{1}, i_{2}\right): i_{1}=i_{2}=i\right.$, $1 \leqslant i \leqslant d+1\}$, we obtain, after the change of variables $u \rightarrow u-x$,

$$
J_{1}(T, \boldsymbol{\theta}, \boldsymbol{y})=\mu^{-1} \sum_{i=1}^{d+1} \sum_{x=T_{i-1}+1}^{T_{i}}\left|\phi_{i}\right|^{\beta} \sum_{u=1}^{T_{i}-x} u^{\beta} P(U=u) .
$$


The inner sum starts at $u=1$ because $T_{i-1}+1-x \leqslant 0$ and $U$ takes only positive values.

Using $\phi_{d+1}=0$ and making the further change of variables $x \rightarrow T_{i}-x$ gives

$$
J_{1}(T, \boldsymbol{\theta}, \boldsymbol{y})=\mu^{-1} \sum_{i=1}^{d}\left|\phi_{i}\right|^{\beta} \sum_{x=0}^{T_{i}-T_{i-1}-1} \sum_{u=1}^{x} u^{\beta} P(U=u) .
$$

We clearly obtain (5.5) when $\beta \leqslant \alpha$. Now assume $\beta>\alpha$. Since $\sum_{u=1}^{x} u^{\beta} P(U=u) \sim$ $\alpha(\beta-\alpha)^{-1} x^{\beta-\alpha} h(x)$ as $x \rightarrow \infty$ and $T_{i}=\left[T y_{i}\right]$, we obtain (5.5) as $T \rightarrow \infty$, with $J_{1}(\theta, y)$ given in the form (5.8). It follows, from the remark after Proposition 5.2, that $J_{1}(\boldsymbol{\theta}, \boldsymbol{y})$ can also be expressed as (5.6). This concludes the proof of Proposition 5.2.

The proofs of Propositions 5.3 and 5.4 are much more delicate. The basic idea is as follows. If

$$
H_{T}(\boldsymbol{\theta}, \boldsymbol{y} ; x, u)=\mu^{-1}\left|\sum_{j=i_{1}}^{i_{2}-1} \theta_{j}\left(T_{j}-x\right)+\phi_{i_{2}}(u-x)_{+}\right|^{\beta} P(U=u-x),
$$

then

$$
J_{\ell}(T, \boldsymbol{\theta}, \boldsymbol{y})=\sum_{\ell_{\ell}} \sum_{x=T_{i_{1}-1}+1}^{T_{i_{2}}} \sum_{u=T_{i_{2}-1}+1}^{T_{i_{2}}} H_{T}(\boldsymbol{\theta}, \boldsymbol{y}, x, u)=\sum_{\ell_{\ell}} \sum_{\mathscr{T}_{T}\left(i_{1}, i_{2}\right)} H_{T}(\boldsymbol{\theta}, \boldsymbol{y}, x, u),
$$

where

$$
\mathscr{T}_{T}\left(i_{1}, i_{2}\right)=\left\{(x, u) \in \mathbb{Z}^{2}: T_{i_{1}-1}+1 \leqslant x \leqslant T_{i_{1}}, T_{i_{2}-1}+1 \leqslant u \leqslant T_{i_{2}}\right\} .
$$

Proceeding as for the term $I$ (see Proposition 5.1), we define 'continuous' versions $\tilde{H}_{T}$ and $\tilde{J}_{\ell}$ of $H_{T}$ and $J_{\ell}$, namely,

$$
\tilde{H}_{T}(\boldsymbol{\theta}, \boldsymbol{y} ; t, s)=\mu^{-1}\left|\sum_{j=i_{1}}^{i_{2}-1} \theta_{j}\left(T_{j}-t\right)+\phi_{i_{2}}(s-t)\right|^{\beta} j(s-t), \quad s, t \geqslant 0,
$$

where

$$
j(u)=P(U>u-1)-P(U>u)=P(U=[u])
$$

and

$$
\tilde{J}_{\ell}(T, \boldsymbol{\theta}, \boldsymbol{y})=\sum_{\mathscr{b}_{\ell}} \int_{T_{i_{1}-1}}^{T_{i_{2}}} \int_{T_{i_{2}-1}}^{T_{i_{2}}} \tilde{H}_{T}(\boldsymbol{\theta}, \boldsymbol{y} ; x, u) \mathrm{d} u \mathrm{~d} x=\sum_{b_{\ell}} \sum_{\mathscr{T}_{T}\left(i_{1}, i_{2}\right)} \int_{x-1}^{x} \int_{u-1}^{u} \tilde{H}_{T}(\boldsymbol{\theta}, \boldsymbol{y} ; t, s) \mathrm{d} s \mathrm{~d} t,
$$

and show that, for $\ell=2$ and $\ell=3$, as $T \rightarrow \infty$,

$$
\tilde{J}_{\ell}(T, \boldsymbol{\theta}, \boldsymbol{y}) \sim J_{\ell}(\boldsymbol{\theta}, \boldsymbol{y}) T^{\beta-\alpha+1} h(T)
$$

and

$$
J_{\ell}(T, \boldsymbol{\theta}, \boldsymbol{y})-\tilde{J}_{\ell}(T, \boldsymbol{\theta}, \boldsymbol{y})=o\left(T^{\beta-\alpha+1} h(T)\right) .
$$


These last two relations are technically involved. We now present some of the details.

Proof of Proposition 5.3. We must establish (5.17) and (5.18) for $\ell=2$. (We may assume that $T$ is so large that $T>\left(y_{i}-y_{i-1}\right)^{-1}$ for all $i=1, \ldots, d$.) We start with (5.17). Fix $\left(i_{1}, i_{2}\right) \in \mathscr{A}_{2}$. Since $u-x$ is bounded away from 0 when

$$
T_{i_{1}-1} / T \leqslant x \leqslant T_{i_{1}} / T<T_{i_{2}-1} / T \leqslant u \leqslant T_{i_{2}} / T,
$$

and since $j(T) \sim \alpha T^{-\alpha-1} h(T)$ as $T \rightarrow \infty$, there is a constant $d_{1}>0$ such that $j(T(u-x)) / j(T) \leqslant d_{1}(u-x)^{-\alpha-1}$. After the rescaling $x \rightarrow x / T, u \rightarrow u / T$, we obtain

$$
\int_{T_{i_{1}-1}}^{T_{i_{1}}} \int_{T_{i_{2}-1}}^{T_{i_{2}}} \tilde{H}_{T}(\boldsymbol{\theta}, \boldsymbol{y} ; x, u) \mathrm{d} u \mathrm{~d} x=T^{2+\beta} j(T) \int_{0}^{\infty} \int_{0}^{\infty} f_{T}(\boldsymbol{\theta}, \boldsymbol{y} ; x, u) \mathrm{d} u \mathrm{~d} x,
$$

where

$f_{T}(\boldsymbol{\theta}, \boldsymbol{y} ; x, u)$

$$
=\mu^{-1}\left|\sum_{j=i_{1}}^{i_{2}-1} \theta_{j}\left(\frac{T_{j}}{T}-x\right)+\phi_{i_{2}}(u-x)\right|^{\beta} \frac{j(T(u-x))}{j(T)} 1_{\left[T_{i_{1}-1} / T, T_{i_{1}} / T\right]}(x) 1_{\left[T_{i_{2}-1} / T, T_{i_{2}} / T\right]}(u)
$$

converges, as $T \rightarrow \infty$, to $\alpha^{-1} H(\boldsymbol{\theta}, \boldsymbol{y} ; x, u) 1_{\left[y_{i_{1}-1}, y_{i_{1}}\right]}(x) 1_{\left[y_{i_{2}-1}, y_{i_{2}}\right]}(u)$, with $H$ defined in (5.7). The dominated convergence theorem applies because $u-x$ is bounded away from zero and, hence, for $T$ large enough, $f_{T}(\boldsymbol{\theta}, \boldsymbol{y} ; x, u)$ is uniformly bounded in $T$. This proves (5.17) for $\ell=2$.

We now turn to the proof of (5.18) for $\ell=2$. Set

$$
J_{2}(T, \boldsymbol{\theta}, \boldsymbol{y})-\tilde{J}_{2}(T, \boldsymbol{\theta}, \boldsymbol{y})=\sum_{\ell_{2}}\left(D_{1}\left(T, i_{1}, i_{2}\right)+D_{2}\left(T, i_{1}, i_{2}\right)\right)
$$

(we suppress the arguments $\boldsymbol{\theta}$ and $\boldsymbol{y}$ ), where

$$
\begin{gathered}
D_{1}\left(T, i_{1}, i_{2}\right)=\mu^{-1} \sum_{\mathscr{T}_{T}\left(i_{1}, i_{2}\right)} \int_{x-1}^{x} \int_{u-1}^{u}\left(\left|A_{T}(x, u)\right|^{\beta}-\left|A_{T}(t, s)\right|^{\beta}\right) j(s-t) \mathrm{d} s \mathrm{~d} t, \\
D_{2}\left(T, i_{1}, i_{2}\right)=\mu^{-1} \sum_{\mathscr{T}_{T}\left(i_{1}, i_{2}\right)}\left|A_{T}(x, u)\right|^{\beta} \int_{x-1}^{x} \int_{u-1}^{u}[j(u-x)-j(s-t)] \mathrm{d} s \mathrm{~d} t
\end{gathered}
$$

and

$$
A_{T}(x, u)=\sum_{j=1}^{d} \theta_{j}\left(T_{j} \wedge u-x\right)_{+}
$$

One can show that there is a constant $d_{2}=d_{2}\left(i_{1}, i_{2}\right),\left(i_{1}, i_{2}\right) \in \mathscr{A}_{2}$, such that

$$
\left.\max _{\mathscr{T}_{T}\left(i_{1}, i_{2}\right)} \sup _{\substack{x-1 \leqslant t \leqslant x \\ u-1 \leqslant s \leqslant u}}|| A_{T}(x, u)\right|^{\beta}-\left|A_{T}(t, s)\right|^{\beta} \mid \leqslant d_{2} T^{(\beta-1)_{+}}
$$

and 
$\sum_{\mathscr{T}\left(i_{1}, i_{2}\right)} \int_{x-1}^{x} \int_{u-1}^{u} j(s-t) \mathrm{d} s \mathrm{~d} t \leqslant \sum_{\mathscr{T}_{T}\left(i_{1}, i_{2}\right)}(P(U=u-x-1)+P(U=u-x)+P(U=u-x+1))$.

Using (5.15), we obtain, for example,

$$
\begin{aligned}
\sum_{\mathscr{T}_{T}\left(i_{1}, i_{2}\right)} P(U=u-x)= & \sum_{x=T_{i_{1}-1}+1}^{T_{i_{1}}}\left[P\left(U>T_{i_{2}-1}-x\right)-P\left(U>T_{i_{2}}-x\right)\right] \\
= & \sum_{x=T_{i_{2}-1}-T_{i_{1}}}^{T_{i_{2}-1}-T_{i_{1}-1}-1} P(U>x)-\sum_{x=T_{i_{2}-T_{i_{1}}}}^{T_{i_{2}}-T_{i_{1}-1}-1} P(U>x) \\
& (\alpha-1)^{-1}\left\{\left(y_{i_{2}-1}-y_{i_{1}}\right)^{1-\alpha}-\left(y_{i_{2}-1}-y_{i_{1}-1}\right)^{1-\alpha}-\left(y_{i_{2}}-y_{i_{1}}\right)^{1-\alpha}\right. \\
& \left.+\left(y_{i_{2}}-y_{i_{1}-1}\right)^{1-\alpha}\right\} T^{1-\alpha} h(T)
\end{aligned}
$$

as $T \rightarrow \infty$ (with the usual interpretation $\left(y_{i_{2}}-y_{i_{1}}\right)^{1-\alpha} \equiv 0$ and $\left(y_{i_{2}}-y_{i_{1}-1}\right)^{1-\alpha} \equiv 0$ when $\left.i_{2}=d+1\right)$. Observe that the factor in braces in (5.24) is non-zero because $\left(i_{1}, i_{2}\right) \in \mathscr{C}_{2}$ (see (4.8)). Hence there is a constant $d_{2}^{\prime}=d_{2}^{\prime}\left(i_{1}, i_{2}\right)$ such that

$$
\sum_{\mathscr{T}\left(i_{1}, i_{2}\right)} \int_{x-1}^{x} \int_{u-1}^{u} j(s-t) \mathrm{d} s \mathrm{~d} t \leqslant d_{2}^{\prime} T^{1-\alpha} h(T)
$$

and therefore

$$
D_{1}\left(T, i_{1}, i_{2}\right)=O\left(T^{(\beta \vee 1)-\alpha} h(T)\right) .
$$

One also shows that there are constants $d_{3}=d_{3}\left(i_{1}, i_{2}\right)$ such that

$$
\max _{\mathscr{T}_{T}\left(i_{1}, i_{2}\right)}\left|A_{T}(x, u)\right|^{\beta} \leqslant d_{3} T^{\beta}
$$

and

$$
\begin{aligned}
\sum_{\mathscr{T}_{T}\left(i_{1}, i_{2}\right)}\left|\int_{x-1}^{x} \int_{u-1}^{u}[j(u-x)-j(s-t)] \mathrm{d} s \mathrm{~d} t\right| & \leqslant \sum_{\mathscr{T}_{T}\left(i_{1}, i_{2}\right)} j(u-x) \int_{x-1}^{x} \int_{\substack{u-1 \\
u-1 \leq t \leq s \leq u \\
u-1 \leqslant s \leqslant}}^{u}\left|1-\frac{j(s-t)}{j(u-x)}\right| \mathrm{d} s \mathrm{~d} t \\
& =o\left(\sum_{\mathscr{T}_{T}\left(i_{1}, i_{2}\right)} j(u-x)\right)=o\left(T^{1-\alpha} h(T)\right)
\end{aligned}
$$

as $T \rightarrow \infty$, since $(s-t) /(u-x)$ is bounded away from 0 in the relevant intervals. Thus,

$$
D_{2}\left(T, i_{1}, i_{2}\right)=o\left(T^{\beta-\alpha+1} h(T)\right) .
$$

as $T \rightarrow \infty$. Relations (5.19), (5.26) and (5.27) imply (5.18) for $\ell=2$, which concludes the proof of Proposition 5.3.

Proof of Proposition 5.4. One must distinguish between the cases $\beta \leqslant \alpha-1$ and $\beta>\alpha-1$. Suppose first $\beta \leqslant \alpha-1$. Then $J_{3}$ (see (5.14)) can be written 


$$
J_{3}(T, \boldsymbol{\theta}, \boldsymbol{y}) \leqslant c \sum_{i=1}^{d}\left(C_{1}(T, i, i+1)+C_{2}(T, i, i+1)\right)
$$

where $c$ is a constant,

$$
C_{1}(T, i, i+1)=\left|\theta_{i}\right|^{\beta} \sum_{\mathscr{T}_{T}(i, i+1)}\left(T_{i}-x\right)^{\beta} P(U=u-x)
$$

and

$$
C_{2}(T, i, i+1)=\left|\phi_{i+1}\right|^{\beta} \sum_{\mathscr{T}_{T(i, i+1)}}(u-x)^{\beta} P(U=u-x) .
$$

But

$$
C_{1}(T, i, i+1)=\left|\theta_{i}\right|^{\beta}\left[\sum_{x=T_{i-1}+1}^{T_{i}}\left(T_{i}-x\right)^{\beta} P\left(U>T_{i}-x\right)-\sum_{x=T_{i-1}+1}^{T_{i}}\left(T_{i}-x\right)^{\beta} P\left(U>T_{i+1}-x\right)\right] .
$$

The first sum in (5.28) is asymptotic to $\sum_{x=1}^{\infty} x^{\beta} P(U>x)=O(1)$ either if $\beta<\alpha-1$ or if $\beta=\alpha-1$ and $\sum_{x=1}^{\infty} x^{-1} h(x)<\infty$, and to $\int_{1}^{x} x^{-1} h(x) \mathrm{d} x$ if $\beta=\alpha-1$ and $\sum_{x=1}^{\infty} x^{-1} h(x)=\infty$. The second sum in (5.28) is bounded by

$$
\sum_{x=0}^{T_{i}-T_{i-1}-1} x^{\beta} P\left(U>T_{i+1}-T_{i}\right)=O\left(T^{\beta-\alpha+1} h(T)\right),
$$

and has the same upper bounds as the first sum. Estimates of the same type hold for $C_{2}$ when $i \leqslant d-1$. This concludes the proof for $\beta \leqslant \alpha-1$.

Consider now the case $\beta>\alpha-1$. We must prove (5.17) and (5.18) for $\ell=3$. Relation (5.17) holds with $\ell=3$, because writing

$$
\tilde{J}_{3}(T, \boldsymbol{\theta}, \boldsymbol{y})=\sum_{i=1}^{d} \tilde{C}(T, i, i+1),
$$

one can show that as $T \rightarrow \infty$,

$$
\begin{aligned}
\tilde{C}(T, i, i+1) & :=\mu^{-1} \int_{T_{i-1}}^{T_{i}} \int_{T_{i}}^{T_{i+1}}\left|\theta_{i}\left(T_{i}-x\right)+\phi_{i+1}(u-x)\right|^{\beta} j(u-x) \mathrm{d} u \mathrm{~d} x \\
& \sim\left[\mu^{-1} \int_{y_{i-1}}^{y_{i}} \int_{y_{i}}^{y_{i+1}}\left|\theta_{i}\left(y_{i}-x\right)+\phi_{i+1}(u-x)\right|^{\beta} \alpha(u-x)^{-\alpha-1} \mathrm{~d} u \mathrm{~d} x\right] T^{\beta-\alpha+1} h(T) .
\end{aligned}
$$

Let us turn to (5.18) for $\ell=3$ and $\beta>\alpha-1$. We are in the case $\left(i_{1}, i_{2}\right) \in \mathscr{A}_{3}$ (see (4.8)), that is $i_{1} \equiv i, i_{2}=i+1,1 \leqslant i \leqslant d$, which is particularly delicate because it implies $T_{i_{2}-1}=T_{i_{1}}$, in the definition (5.15) of $\mathscr{T}_{T}\left(i_{1}, i_{2}\right)$. The estimation (5.23) is still valid but (5.24) fails, because now (5.23) converges to the constant $\mu$, and hence one obtains, as $T \rightarrow \infty$, 


$$
D_{1}(T, i, i+1)=O\left(T^{(\beta-1)_{+}}\right)
$$

One also shows that

$$
D_{2}(T, i, i+1)=o\left(T^{\beta-\alpha+1} h(T)\right),
$$

which concludes the proof of Proposition 5.4.

\section{Stationarity of the increments}

In this section, we use the structure of the (finite-dimensional) characteristic function (2.11) of $Z_{\beta}$ to show that this process has stationary increments. Observe that $(2.11)$ defines the finite-dimensional distributions of the process $Z_{\beta}(y)$ for all $y \geqslant 0$.

Proposition 6.1. The processes $\left\{Z_{\beta}(y), y \geqslant 0\right\}$ have stationary increments.

Proof. We have to show that

$$
\left\{Z_{\beta}(y+h)-Z_{\beta}(h), y \geqslant 0\right\} \stackrel{\mathscr{D}}{=}\left\{Z_{\beta}(y)-Z_{\beta}(0), y \geqslant 0\right\}
$$

for all $h>0$. Since $Z_{\beta}(0)=0$, it is sufficient to show that for any real $\theta_{1}, \ldots, \theta_{d}$,

$$
\sum_{j=1}^{d} \theta_{j}\left[Z_{\beta}\left(y_{j}+h\right)-Z_{\beta}(h)\right] \stackrel{\mathscr{Q}}{=} \sum_{j=1}^{d} \theta_{j} Z_{\beta}\left(y_{j}\right) .
$$

We will prove equality of the scale parameters. Since this is clearly the case when $\beta \leqslant \alpha$ (see (2.12)), we can suppose $\beta>\alpha$.

The $\beta$ th power of the scale parameter of the left-hand side of (6.1) can be written $I(\boldsymbol{\theta}, \boldsymbol{y}, h)+J(\boldsymbol{\theta}, \boldsymbol{y}, h)$, where

$$
\begin{gathered}
I(\boldsymbol{\theta}, \boldsymbol{y}, h)=\int_{0}^{\infty}\left|-\phi_{1}(h \wedge x)+\sum_{j=1}^{d} \theta_{j}\left[\left(y_{j}+h\right) \wedge x\right]\right|^{\beta} x^{-\alpha} \mathrm{d} x, \\
J(\boldsymbol{\theta}, \boldsymbol{y}, h)=\int_{0}^{\infty} \int_{0}^{\infty}\left|-\phi_{1}(h \wedge u-x)_{+}+\sum_{j=1}^{d} \theta_{j}\left(\left(y_{j}+h\right) \wedge u-x\right)_{+}\right|^{\beta} \alpha(u-x)_{+}^{-\alpha-1} \mathrm{~d} u \mathrm{~d} x
\end{gathered}
$$

and $\phi_{1}=\sum_{j=1}^{d} \theta_{j}$. We thus have to show that

$$
I(\boldsymbol{\theta}, \boldsymbol{y}, h)+J(\boldsymbol{\theta}, \boldsymbol{y}, h)=I(\boldsymbol{\theta}, \boldsymbol{y}, 0)+J(\boldsymbol{\theta}, \boldsymbol{y}, 0) .
$$

Starting with the $I$-term, make the change of variables $x \rightarrow x-h$ to obtain

$$
I(\boldsymbol{\theta}, \boldsymbol{y}, h)=\int_{-h}^{\infty}\left|-\phi_{1}(h \wedge(x+h))+\sum_{j=1}^{d} \theta_{j}\left(y_{j} \wedge x\right)+\phi_{1} h\right|^{\beta}(x+h)^{-\alpha} \mathrm{d} x .
$$

Write $\int_{-h}^{\infty}=\int_{-h}^{0}+\int_{0}^{\infty}$ and note that $\int_{-h}^{0}=0$, while the integral $\int_{0}^{\infty}$ yields 


$$
I(\boldsymbol{\theta}, \boldsymbol{y}, h)=\int_{0}^{\infty}\left|\sum_{j=1}^{d} \theta_{j}\left(y_{j} \wedge x\right)\right|^{\beta}(x+h)^{-\alpha} \mathrm{d} x
$$

Let us now turn to the $J$-term and make the change of variables $u \rightarrow u-h, x \rightarrow x-h$. We obtain

$$
J(\boldsymbol{\theta}, \boldsymbol{y}, h)=\int_{-h}^{\infty} \int_{-h}^{\infty}\left|-\phi_{1}(0 \wedge u-x)_{+}+\sum_{j=1}^{d} \theta_{j}\left(y_{j} \wedge u-x\right)_{+}\right|^{\beta} \alpha(u-x)^{-\alpha-1} \mathrm{~d} u \mathrm{~d} x
$$

since, for example, $\left(y_{j}+h\right) \wedge(u+h)-(x+h)=y_{j} \wedge u-x$. Now write

$$
\int_{-h}^{\infty} \int_{-h}^{\infty}=\int_{-h}^{0} \int_{-h}^{0}+\int_{0}^{\infty} \int_{-h}^{0}+\int_{0}^{\infty} \int_{0}^{\infty}+\int_{-h}^{0} \int_{0}^{\infty}
$$

and note that the first two integrals are identically zero. The third integral equals $J(\boldsymbol{\theta}, \boldsymbol{y}, 0)$, while the fourth is

$$
\begin{aligned}
\int_{-h}^{0} \int_{0}^{\infty}\left|\phi_{1} x+\sum_{j=1}^{d} \theta_{j}\left(y_{j} \wedge u-x\right)\right|^{\beta} \alpha(u-x)^{-\alpha-1} \mathrm{~d} u \mathrm{~d} x & \\
& =\int_{0}^{\infty}\left|\sum_{j=1}^{d} \theta_{j}\left(y_{j} \wedge u\right)\right|^{\beta} \mathrm{d} u \int_{-h}^{0} \alpha(u-x)^{-\alpha-1} \mathrm{~d} x \\
& =\int_{0}^{\infty}\left|\sum_{j=1}^{d} \theta_{j}\left(y_{j} \wedge u\right)\right|^{\beta}\left[u^{-\alpha}-(u+h)^{-\alpha}\right] \mathrm{d} u \\
& =I(\boldsymbol{\theta}, \boldsymbol{y}, 0)-I(\boldsymbol{\theta}, \boldsymbol{y}, h) .
\end{aligned}
$$

This establishes (6.2) and proves that $Z_{\beta}$ has stationary increments.

\section{Acknowledgements}

The second author was partially supported at Boston University by National Science Foundation grants DMS-9404093 and NCR-9404931.

\section{References}

Leland, W.E., Taqqu, M.S., Willinger, W. and Wilson, D.V. (1994) On the self-similar nature of Ethernet traffic (Extended version). IEEE/ACM Trans. Networking, 2, 1-15.

Levy, J. and Taqqu, M.S. (1987) On renewal processes having stable inter-renewal intervals and stable rewards. Ann. Sci. Math. Québec, 11, 95-110.

Mandelbrot, B.B. (1969) Long-run linearity, locally Gaussian processes, H-spectra and infinite variances. Internat. Econom. Rev., 10, 82-113. 
Samorodnitsky, G. and Taqqu, M.S. (1994) Stable Non-Gaussian Processes: Stochastic Models with Infinite Variance. London: Chapman \& Hall.

Taqqu, M.S. and Levy, J. (1986) Using renewal processes to generate long-range dependence and high variability. In E. Eberlein and M.S. Taqqu (eds), Dependence in Probability and Statistics, pp. 73-89. Boston: Birkhäuser.

Taqqu, M.S., Willinger, W. and Sherman, R. (1997) Proof of a fundamental result in self-similar traffic modeling. Comput. Commun. Rev., 27(2), 5-23.

Willinger, W., Taqqu, M.S., Sherman, R. and Wilson, D.V. (1997), Self-similarity through highvariability: statistical analysis of Ethernet LAN traffic at the source level. IEEE/ACM Trans. Networking, 5(1), 71-86. Extended Version of the paper with the same title that appeared in 1995 in Comput. Commun. Rev., 25, 100-113.

Received May 1997 and revised May 1998 